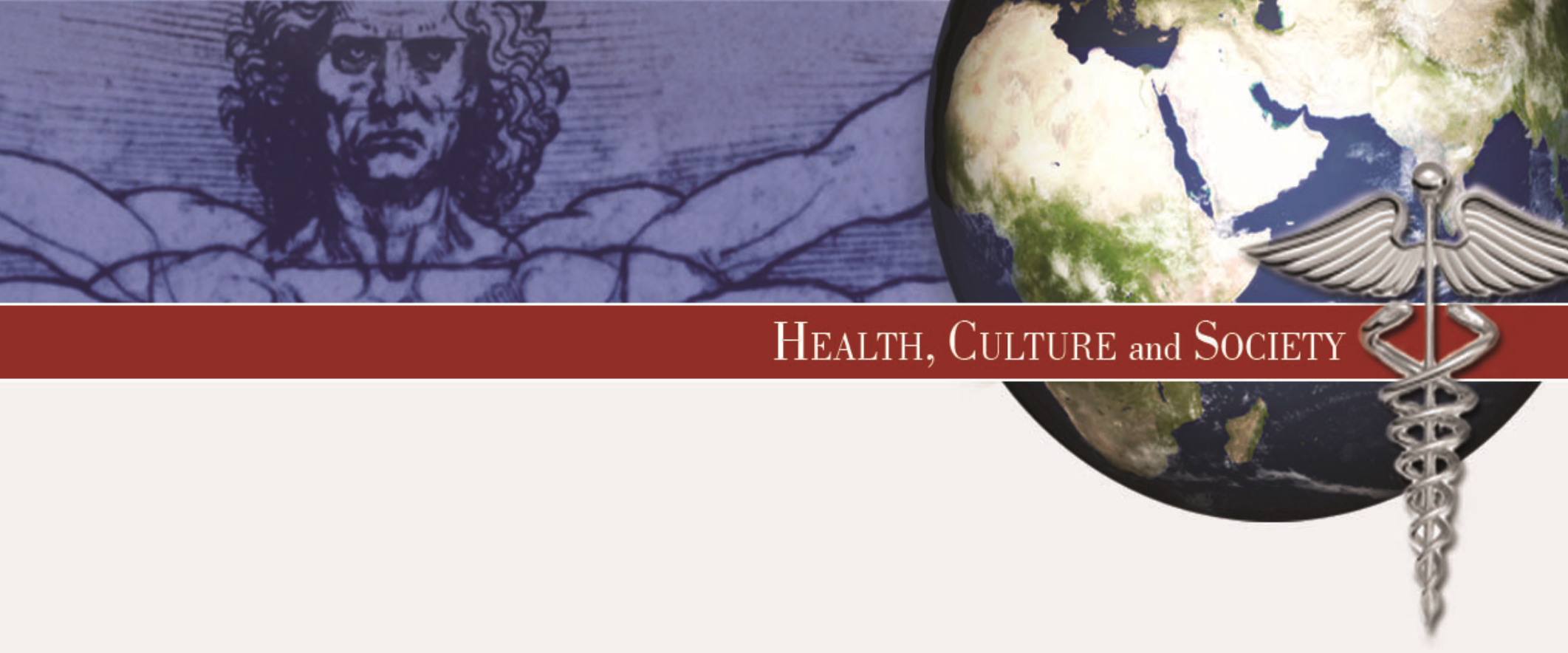

\title{
The Identity Work and Health of Intensive Motherhood
}

\section{Paltineau}

Volume 3, No. 1 (2012) ｜ ISSN 2161-6590 (online) DOI 10.5195/hcs.2012.101 | http://hcs.pitt.edu

\section{(c)) EY-NC-ND}

This work is licensed under a Creative Commons Attribution-Noncommercial-No Derivative Works 3.0 United States License.

\section{ULIS D-Sonte}

This journal is published by the University Library System of the University of Pittsburgh as part of its D-Scribe Digital Publishing Program, and is cosponsored by the University of Pittsburgh Press. 


\begin{abstract}
The French government strictly frames the health of mothers, establishing specific protocols of maternal healthcare during pregnancy and childbirth, as well as for baby care.

Intensive mothers seek to free themselves from a state controlled health care system, and seek to undertake their own therapeutic of pregnancy on the fringes of standardized procedures. Their behaviors enable them to operate an identity work as mothers, and as intensive mothers in particular. These women, use health as a tool to construct their identity as an individual, and, likewise, as part of a couple and a group.

But what characterizes the health choices of intensive mothers and what are the hallmarks to intensive motherhood? How is a specific parental identity formed? And how, moreover, do more natural health behaviors enable intensive parents to assert themselves as individuals within an everchanging society?
\end{abstract}

Keywords: intensive motherhood; mothering; childcare; parenthood; childbirth

Health, Culture and Society 


\title{
The Identity Work and Health of Intensive Motherhood
}

\author{
M. Paltineau ${ }^{1}$
}

\section{Introduction}

During the 20th century, France had sought to progressively attend to pregnancy and labor with medical care. Where these realities of were once handled by women, inside the home, in the last century hospitals soon became the place for childbirth. Healthcare centers have today become the reference point for prenatal, postnatal and follow-up care. In 2010 it was reported that French women had on average five ultrasounds during their pregnancy and $45 \%$ underwent at least ten prenatal medical visits. $97 \%$ of the births occurred at hospital facilities (Blondel \& Kermarrec, 2011).

Yet despite the growth of services, centers and patients attended to, a category of people do not accept such forms of medicalization. This trend, is called intensive motherhood or intensive parenting, both, terms which have been developed in the USA by the sociologist Sharon Hays (1998) referring to several practices and representations through which the mothers (and sometimes the fathers) overplay their parental roles. Such commitment is typical of educated women of the middle and upper classes: it is part of a larger societal context, which goes against the current consumer tendencies of our society. Our present economic crisis plays a role in the development of intensive motherhood, since it urges people to rethink what really matters to them, socially, materially, spiritually and historically. The era of capitalism and mass consumption appears an outdated and saturated reality, and more attention is given to well-being, individuality and family than to material comfort alone. In this context, the interest for the health of mothers and children is growing; it is actually the heart of the intensive motherhood trend and defines the identity of intensive parents.

No woman is an intensive mother per se; intensive motherhood is an ideal type, corresponding to a range of behaviors and practices adopted by an increasing number of women, even partially so. The study of this phenomenon is relevant not only because such a trend is observable in large portions of the French middle and upper classes, but also because it is to be found in many other occidental societies.

This trend thus enables us to analyze the new social conditions of becoming a parent, and the way healthcare is articulated institutionally. Intensive motherhood undeniably questions the role of both government and hospital regarding pregnancy and childbirth, since the legitimacy of certain medical practices and the tradition of the omniscient physician are no longer accepted. This trend attracts new qualified health workers, and gives subjective importance to alternative techniques. In the private sphere, practices change too and naturalist tendencies are increasingly chosen over allopathic interventions.

$1 \quad$ EHESS: I'École des Hautes Études en Sciences Sociales 
What this above all signals is a strong identity assertion: intensive motherhood is a conscious process, and it is identifiable as an element of feminine emancipation, as paradoxical as it may be at first sight. Identity is the core of our considerations when analyzing a trend where each woman and man realizes what matters to them, and where they become the actors of every single choice they make. Sociological research supports this. Harrison White's approach (1992), which focuses on the meaning of actions, asserts that the identity concept is equivalent to the concepts of actor or agent found in other sociology theories: identity designates "any source of action that cannot be explained by biophysical regularities, and to which observers can give a meaning" (White, 1992, p.35). White further emphasizes that an action becomes subjective as soon as an individual allots meaning to it; the fulfilling of this action enables the individual to assert his identity. The concept of actor, moreover, comes from the notion of identity and is formed by it, for when the individual acts with consciousness (i.e. when he is an actor), he/she generates his/her own particular identity. Identity thus focuses on a permanent work undertaken in order to maintain individual coherences.

The author carried out in depth empirical research composed of in situ observations in hospitals and birth centre facilities and seventy longitudinal interviews with intensive parent types and health care professionals. The analysis of this empirical research puts into perspective the relationship between health, individuality and collective identity: what impacts, for example, does one's health path have upon identity assertion? Are pregnancy and labor choices tools for asserting, and indeed constructing, identity? How does a pregnant woman's healthcare define maternal identity in general, and that of intensive mothers, particularly?

The issue of power and gender struggles between the medical community and health care users makes us understand how intensive motherhood shapes a new identity for women and mothers, and how it makes them become individuals who are responsible for their actions. By refusing excessive medicalization, this trend can be seen to generate a critical reflection on the links between technique and identity: how, for example, do mothers' health paths favor identity assertion? And what collective identity emerges with the new and challenging practices defining the direction of this trend?

This research review article presents three main stages of maternity health paths: pregnancy, labor, and post-partum. For each of these stages, the author analyzes typical practices of intensive motherhood, and how they enable individual assertion in the French healthcare system.

\title{
II. Reinvesting in Pregnancy
}

\begin{abstract}
You shape your own history, these decisions, you will make them with [the midwives]. (...) here, we are a place to exchange, to listen, to speak. We are with someone who gives you the tools to make the right decision (...) at the CALM, here, at every medical visit, the project develops. You talk about it, you live it. (Audrey, at the birth center CALM, Paris)
\end{abstract}

In the social groups under scrutiny, pregnancy and childbirth are considered as natural events. They do not request a systematic hospitalization, but only care, emotional support and the continuous presence of a healthcare specialist (not necessarily a physician). This ideology is borne from the philosophy developed in the 1970s by Michel Odent and Frédéric Leboyer, who defended a naturalist vision of maternity, criticizing pathological understandings and the dominion of the biomedical model. The method of soft childbirth, which focuses on the emotive disposition of the baby, what the baby feels, emphasizes the softness of approach in medical care and the ambience: half-lighting, touch, and immediate and long contact to the mother after birth. Proponents of this express that French society was lost in an international race for medical techniques, losing sight of the humanity in the name of medical procedure. This is partially explainable by the loss of popular know-how, the lack of intergenerational transmission, and the heavy

The Identity Work and Health of Intensive Motherhood Volume 3, No. 1 (2012) | ISSN 2161-6590 (online) | DOI 10.5195/hcs.2012.101 | http://hcs.pitt.edu 
incursion of medical practitioners into the childbirth. What this further evidences is that the dichotomy between technique and interpersonal boundary is strong, and that the methods employed by hospitals fail to engage with these atmospheric dimensions effectively. Thus the need for new methods and philosophies of childbirth (Jacques, 2007).

Following up this trend which was launched in the 1970s, pregnant women from middle and upper classes have made choices that increasingly moved away from standardized pregnancy paths and in doing this they consciously contravened standard protocols, not adhering to the suggested paths of healthcare institutions. Such mothers stood back from the choices they were given, and seldom chose the classical ways. In a radical move they questioned the involvement of medical techniques and healthcare professionals in their own histories, and they sought to question the significance and meaning of each procedure and intervention in terms of the integrity of their bodies and identities.

If intensive mothers usually undergo the prescribed ultrasounds, the trend is to refuse to know the baby's gender before the birth, considering that some medical techniques are unnecessary. Even when intensive mothers go to the medical appointments, some exams are systematically refused: amniocentesis and systematic vaginal examination for example (the former because of miscarriage risk, the latter because it is considered as a violation of women's intimacy when conducted repeatedly). Intensive mothers claim the right to own their body, and they do not want to be deprived of this right by medical practice.

This lack of comfort and this misunderstanding lead mothers to seeking skilled health care outside of the hospital. Today, an increasing number of French women call on independent midwives for their pregnancy follow-up and antenatal classes. Indeed, intensive mothers escape antenatal group classes organized by hospitals, and they favor individual or small group classes led by independent midwives: the intensive mother believes her intimacy and personal history is greater respected and valued in a more personalized setting. This again, flagging the distinction between a therapeutic atmosphere and an institutional environment.

These freelance midwives usually carry out one-to-one care: the same midwife takes care of a mother from the outset of pregnancy until the weeks immediately following birth. In this context, the human relationship, the bond that is, between mother and the midwife is crucial, as it is able to generate the personal development of every woman. One-to-one care is considered as an alternative follow-up, based on "human and relational qualities combined with skills. It is considered as a factor of risk prevention, and it favors the physiological phases of maternity" (Interview with a midwife at the birth center CALM, 2010).

Thus, independent midwives are the identifiable partner for intensive mothers' pregnancy follow-ups, their closeness with the mothers being favored by their practices, their gender, and the fact that they attend in the home or in small practices rather than in large hospitals.

In addition to pregnancy monitoring, intensive mothers often opt for other types of additional prenatal care. Several options are available, with different healthcare specialists. To name only those mentioned by our interview subjects, haptonomy, relaxation therapy, antenatal singing, and antenatal yoga featured significantly. Indeed, each of these specialist fields mutually offers a personalized pregnancy follow-up giving each mother the opportunity to commit into her own health and that of her child. This commitment occurs in different ways: by finding the matching therapy, by doing it continuously over a long period of time, and by being staunch with its utility. These new practices enable mothers to enter specific social circles, the encounters and exercises they do being a first step into their identity assertion and their intensive motherhood.

These practices inevitably make new actors intercede, who complete or replace the roles of physicians and midwives: doulas, reflexologists, antenatal singing teachers, antenatal yoga teachers. These actors shape the world of intensive motherhood furnishing it with unique dynamics where new relationships, exchanges and meanings emerge

The Identity Work and Health of Intensive Motherhood Volume 3, No. 1 (2012) | ISSN 2161-6590 (online) | DOI 10.5195/hcs.2012.101 | http://hcs.pitt.edu 
outside of hospital walls. Intensive parents avoid hospital facilities (even if they do not refuse it as a whole), and build a parallel universe where different actors stand for the health of pregnant women and their children.

The newest element brought by these dynamics is the fact that healthcare providers are considered to be on equal footing with pregnant women: they back them in their choices, without claiming to have knowledge that mothers do not have. Given their education and their habit of seeking and researching the information they need, intensive mothers do not always have the same knowledge as their health partners. The goal of these professionals is to stay by the side of the mothers, precisely to accompany mothers, to let them be a better version of themselves and find a harmony with their life principles and philosophies. Women are thus able to assert their identities, instead of being passive towards their pregnancies. In this respect, intensive mothers have the feeling of participation and accompaniment with such forms and methods of follow-up, wherein exists a larger action range than in hospitals. .

Another distinctive element to intensive pregnancy is the way in which women have considered their work role: immediately after discovering that they are pregnant, women will be quick in pushing their jobs into the middle ground, and by allotting less symbolic significance and time to their work, early maternity leave becomes a favorable option. This commonplace occurrence is a telling sign that focus is directed to health and the health of the baby to come.

This is where the first act of identity assertion emerges. These women seek to protect their health and that of their children, by focusing on corporeal self-listening, self-knowledge, respect and consciousness this enveloped by the council of others, communication skills, and medical knowledge. Mother identity is shaped during the pregnancy, as women define what who they want to be and what impulse they want to give to their maternity. Women are increasingly asked to assert through their motherhood, and to consider it as the primary factor in developing and shaping their identity: this is the so-called "mothering as identity work" (Faircloth, 2009).

As soon as a woman chooses to step away from classical healthcare paths, she undertakes an identity work: refusing the standardization of care during the pregnancy means that you have thought about who you want to be, and that you have consciously decided to be different. Those interviewed claim their choices as guarantors of an individual freedom; they assert their subjectivity and become actors of pregnancy and birth. This process is enabled by a long personal research, deep self-reflection on pregnancy, and often through peer-exchanges.

Our analysis was based on sociological interaction theories, according to which interactions with the rest of the society enable intensive parents to claim their choices in a general and unconditional way. Monique Bydlowsk (2005), for example, speaks of pregnancy as a "maturation crisis", a time of self probation; François Dubet and Danilo Martucelli likewise assert the importance of subjective experience: "because he/she wants to be an actor, individuals tends to see themselves as the authors of their lives" (Dubet, 1998, p. 78). The individual feels responsible for the meaning given to his/her existence, and pregnancy in particular is a strong, defining moment in which individual subjectivity has the opportunity to crystallize, for the father as well as the mother.

\section{Making Childbirth Your Own}

The idea, here, is just that everything you experience satisfies you, and meets your needs. You are the ones who decide about what you want for yourself, your baby, your partner..." (Audrey, at the birth center, CALM, Paris).

In France, women can give birth in a health facility (a hospital or a clinic, private or public), in facilities assimilated to birth centers, or at home. $97 \%$ of births, however, occur in a hospital setting, and for good reason,

The Identity Work and Health of Intensive Motherhood

Volume 3, No. 1 (2012) | ISSN 2161-6590 (online) | DOI 10.5195/hcs.2012.101 | http://hcs.pitt.edu 
because pregnancies and deliveries are highly standardized and extremely medicalized. Since the 1980s, the French government has invested in a project of controlling pregnancies and childbirths through the national healthcare system, in order reduce both child and mother mortality. The French medical community displays numerous technological and organizational innovations, and the French state invests substantially in obstetrics, with the precise aim to reduce risks.

For these reasons, the formalities of delivery in a hospital setting give very little space to individual initiative in a service of highly codified practices and by heavy procedures. Parents are confined to the role institution has set for them; French obstetrical culture advocating an interventionism that is proportional to the safety level of the hospital facility (following French law, there are three categories of maternity wards). The "laissez-faire" attitude does not exist much in French hospital facilities where the pregnant woman is monitored, on the drip, and must generally keep the gynecologic position, at least during the final stages of labor. She also has to accept any decision made for her by the medical team, whether they are causing the childbirth, calling the physician, using forceps, performing an episiotomy or a caesarian section. All these practices, and many more, are carried out under the guise of technique, and health control. Physicians and midwives are the holders of this knowledge, and they are the ones who regulate the labor and give it a special rhythm. Mothers, amongst this flurry of technique and procedure, are passive: they often do not know what is happening, the details of the procedures are not explained, and they cannot give their opinion. The Parturient is often distraught, thrown into a universe they do not always know well, and they have no other choice but to accept the situation, even if they may bitterly regret it afterwards. When they look back, intensive mothers often can see the inferior position they held during labor.

Within this context, the hyper-medicalization of labor may be criticized, and hospitals may be accused of dehumanization. It is none too rare that the largest maternity wards are often reproached for the lack of care and listening; the medical act is executed submissively, and the symbolic violence of this cold, technical and mostly masculine world becomes a reality to be questioned. Since the 1970s, several feminists, ecologists and philosophers have denounced what appeared to be a uniquely French "centrism" of hospitals. Ivan Illich and Michel Foucault, for instance, took up arms against the perverse effects of biotechnologies, which they held responsible for the loss of meaning of motherhood, the fragmentation of corporeal integrity, the excess of tools, and lastly, the eviction of the individual from the human sphere. In the light of this, we would do well to remember the work of François Dubet (1998, p. 71) which highlights the "poverty of the interpersonal dimension" to be found in hospitals, where technique reduces maternity to its biological aspects, aversely pushing away all other human and cultural dimensions.

Thus, after decades of developing system of assistance where medicalized childbirth is the norm and protocol of procedure, certain thinkers have tried to show that a respectful childbirth does not always imply an excessive use of medical technique. If hospitals still tend to focus on heavy and standardized protocols, out-of-hospital births are now a trend, in line with new social assertions of the Self, the body and identity. Intensive mothers realize that they can give birth in other facilities; they want to assert their choices, take control of their labors, and enforce their individuality. Intensive parents want to bring the birth outside of the hospital, because they believe that it is a private issue, which does not have to involve modern healthcare and technologies, except in the very rare cases of problems and complications.

For intensive parents, the risk dynamics are reversed: unlike the representation that considers the hospital as the safest place to give birth, intensive parents sooner consider that risk is the total and complete dependence on healthcare providers, and the loss of personal reflection within the childbearing process. In this respect, the principle of non-intervention prevails. These reflections consequently lead to a growing interest for birthing centers and home births. 
Birthing centers are yet to become commonplace on the French landscape since they are still attached to hospitals. Labor takes place in the so-called birth center, but the delivery must occur inside the hospital, close to the medical team. This is regulated by the principle of risk prevention, which the French government does not want to be flexible with. Nevertheless, in recent years, impassioned voices calling for the independence of these birth facilities have emerged. Birthing centers respond to the demand to diversify healthcare facilities, according to the instructions of the WHO, which asks for caution towards over-medicalization, and stands up for women's and families' free choice about where they want to give birth: "Every woman has the right to discuss her concerns in an environment in which she feels confident", and, "A woman (or her family if necessary) should give informed consent before the provider performs any procedure" (WHO, 2003). Women who go for this kind of birth facility can be considered as intensive mothers, since their approach is defined by a quest for the most personalized and respectful childbirth possible.

Hospitals, under such social pressures, are making efforts to facilitate more natural births, respecting the freedom of the parturient, and providing her with several options for the labor. Hospitals thus now tend to soften standardized procedure, in order to allow every woman to feel unique, and to have a customized delivery. Thus the attempt to combine medical technique with personalized care, signals that hospitals are increasingly conscious of that fact that women who seek alternatives to over-medicalized practices are able to give birth in good conditions, without risks. These women wish to overcome medical imperatives to better experiment the childbirth and thus better own it, are generally intensive mothers, who are able to complete (or replace) technical medical care, thanks to the knowledge they have accrued about their bodies and childbirth. In this respect, more and more French hospitals have multipurpose technical facilities with an emphasis on ambience, i.e. fully equipped delivery rooms: balloons, chairs, bathtubs, etc.

Intensive mothers seek for the meaning of childbirth. They consider their maternity as a mission they have to accomplish. They want to be the actresses of this event, suffering when the labor process has been taken from them. Indeed, the identity of motherhood starts to be shaped during pregnancy and fully develops during the birth phase: mothers feel invested with the mission of giving birth to a new human being, and they suffer when deprived of this ability (for example when physicians use forceps, or worse, during caesarean sections). To not have control during the birth can thus contravene important stages of identity work and self assertion.

It is important to note that the pain experience during birth has an initiatory value: it strengthens the mother and generates a new individual. Delivery is often presented as a double birth: the baby's birth, but also the mother's birth, for who the experience of pain plays a role on her individual identity. The childbirth experience is enhanced, the inner feelings provided by the process of establishing oneself as a mother are promoted, and the status that the delivery pain give to mothers is preserved: feeling the delivery and having the ability to give birth play a role in the development of self-esteem - controlling one's body is a particularly enhancive personal achievement.

Women can become the actresses of childbirths when they are able to make the decisions, or when they know and understand what is happening. Understanding and knowledge are the keys to action and assertion, where each experience is enhanced in its uniqueness, and the multiplicity of choices and life stories are highly appreciated. Birth, in this way, holds a distinctly biographical value for the mother. Home birth is yet another way to move births outside of hospital walls and institutionalized practices, yet remains on the fringes of French society. Within the home setting, the birth is accompanied by the midwife who undertook the one-to-one care, and, at times, a doula. This practice remains relatively unknown, stigmatized, somewhat taboo, and only represents $0,5 \%$ of French births. The mother who opts for such a birth is often considered outlandish in her decision, yet greater reflection would reveal that they are actually intensive parents, who precisely choose a home birth for reasons of principle and conviction. The mother's and child's health is what holds importance for the home-birth mother for whom the hospital does not provide the necessary care (or rather an excess of unnecessary care), in order to guarantee the good health of the mother and her child. For the mother who chooses home birth, the risk is precisely the hospital environment.

The Identity Work and Health of Intensive Motherhood Volume 3, No. 1 (2012) | ISSN 2161-6590 (online) | DOI 10.5195/hcs.2012.101 | http://hcs.pitt.edu 
Intensive mothers who opt for home birth often make this choice having previously experiencing hospital birth. They now want to be in charge of their labor through a personal research and heightened knowledge of delivery procedures, including sometimes very precise technical knowledge. Such surfeit of information gives the mother a self confidence and makes the notion of risk relative. During home births, parents are no longer laypeople, but they stand on the side of science and responsibility. ${ }^{2}$

People who gave birth at home consider it as a life-changing, biographical, event, which enables them to full assertion, this contributing to the identity of both parents. By refusing the coverage of their delivery by hospital and state, and by seeking alternative and informed paths, they stand on the fringe of classical medical paths, and differentiate themselves from the norm of natural scientific medicine. By stepping away from social norms, they, both parents, shape their parenthood with alternative practices. Thus the at times fringe identity of intensive parents is that of a creative and concerted responsibility rooted in a constructive health choice, where the child is delivered into an ambience, and not merely a clinical environment. These alternative childbirths belong to the intensive parenthood commitment, which develops further after the birth, throughout primary and successive years of childcare, where the father, actively participates in the forming and sharing of values.

Indeed, alternative childbirths enable the father to find a closer place by the side of the mother and the child. Within the hospital setting, they are the audience to record the event, encourage and jeer on the mother the best they can, despite their lack of understanding of the situation. Contrastingly, during natural childbirths, parents actively prepare together: intensive fathers generally participate in antenatal classes, and according to the chosen technique, the father is asked to support the mother in an active way (for example with the haptonomy): intensive motherhood generates an energy that brings the couple together, and the intensive parenting approach is shared.

\section{Intensive Mothering}

My midwife took care of it, so we were not separated; she put it on my stomach straight away, and she respected what we agreed on. But I imagine that if I had been in a hospital facility, without my midwife, it would not have happened in the exact way I wanted it to. (Victoire, 14/06/2011)

From the very first moments after the birth, intensive mothers distinguish themselves by the care they give to the newborn. Indeed, they control the care it receives, refusing the excess of medical examinations for example, the cleaning of its respiratory tract. Rather, the intensive mother seeks to keep the baby close to her chest, making a point of maintaining "skin to skin" contact. Breastfeeding is particularly enhanced: the first feeding is important for the intensive mother, since it provides nutritive elements the baby needs to survive and to develop properly. Often, medical imperatives will interfere with these very first moments of intense sensitivity and contact and mothers will

\footnotetext{
${ }^{2}$ One of our interviewed mothers gave birth to her child alone, without any assistance other than that of her husband at the very end of the delivery. To prepare for this homebirth, she consulted numerous books designed for midwives and nurses, in addition to diverse material on motherhood. This is a telling example of how a person can shape her destiny, can assert herself, and surpass herself through intensive motherhood. Several networks of intensive parents develop, which enable them to connect and discuss about their home birth experiences. This actually confirms our hypothesis according to which intensive parenthood creates new social networks, enabling a collective identity assertion outside of official facilities.
} 
voice refusal of any separation from their babies including certain medical exams. They request that procedure is undergone in the same room, if possible with the baby still close to the chest. Intensive mothers, in asserting their identity at the same time as care for the child, seek to promote a knowledge of motherhood, one, that is at times, parallel to that of the physician. It is of worth to note that momentum of identity assertion following birth: if mothers are sometimes manhandled during labor, or if they feel that the birth was not under their control, the situation then changes is reversed once the baby is born. Certain women express suffering during delivery, but a post-natal strength to assert choices. This demonstrates that if intensive mothers acknowledge certain legitimacies of healthcare professionals during delivery, they want to take over the baby care immediately afterwards. It is generally accepted that physicians know which decision to make during delivery, however, intensive mothers consider themselves to be in the best position to decide about baby care. In this respect, medical knowledge seems harder to obtain than nursery nursing knowledge.

Health is thus perceived as a combination of scientific and secular knowledge, and if for the delivery, medicine prevails, the secular or instinctive knowledge (as some subjects call it) is enough for nursery nursing, a care which flows from the mother forming the mother-child bond. This said, intensive mothers are often at odds with nursery nurses regarding relevant and procedural child-care: intensive mothers have read both broadly and specifically and they generally trained and cultivated a mother's knowledge with their older children. A mother will commonly know, for example, how to bath a baby, how to change a diaper; but the main issue of breastfeeding remains. In France, women who have just given birth have to hold record of each feed. Intensive mothers always breastfeed on demand. In such a context, record keeping is difficult, as breastfeeding is almost continuous. As a result, a conflict in practice can with healthcare professionals, who will sooner blame mothers for not knowing how to care for their children properly. An intensive mother will claim the right to breastfeed their babies the way they know, without necessarily receiving the approval of medical workers. Here, experience can shake the principles of theory, and a mothering practice can contravene hospital protocol. The distinction of status, role and function of both, that is, the medical worker and the mother, thus enter into tension.

It is thus not unusual, given the possibility of such tension, that intensive mothers feel uncomfortable and under pressure while in hospital, resulting in a shortened stay at the maternity ward. Such an identity assertion is expressed by the act and decision of an early home return: the mother asserts independence of the hospital institution and her subjectivity asserts an active persona in the drama of motherhood. Logically, such an assertion often generates conflicts at the level of physician intervention and hospital administration: with the child's health at possible stake, the hospital does not allow any right to intervene, and considers that the new born is under its responsibility and decision. Yet, through conflict of ownership and responsibility, the mother will assert final judgment. The precise tension is thus: (1) mothers have an endogenous point of view, according to which health comes from the individual and should be maintained by the mother's care and warmth and ambience of the home;(2) physicians embrace an exogenous point of view about health: it is not a priori granted, but is generated by the external intervention of the health practitioners. And where mothers have the subjective experience, and knowledge, of their child's health, it is still, nevertheless, a public matter for healthcare professionals.

This confrontation between the public and the private sphere tends, however, to resolve into a common practice when the family returns home, where the practice of intensive mothering continues, by both parents, for a certain period, generally until the child is of school attending age. As we have reflected upon, intensive mothers distinguish themselves by their behavior during the first weeks following birth: the mother will not remain bed-bound, and perineal rehabilitation becomes a personal responsibility, while other mothers refer to medical workers. This was not an unusual habit a century ago, but it has been abandoned in the name of modernity and feminine emancipation. Intensive mothers sooner combine modernity and tradition, and thus create new behavioral schemes, that we could call postmodern, and in this particular health specific social context, postmodern motherhood.

The Identity Work and Health of Intensive Motherhood Volume 3, No. 1 (2012) | ISSN 2161-6590 (online) | DOI 10.5195/hcs.2012.101 | http://hcs.pitt.edu 
Intensive mothers will seek to adapt and redefine the ancient with the modern practice against the background of a shifting society, The adaptation and redefinition of traditions, is all in the name of the child's benefit, as the guarantee and measure of health, diet, parental bond, and psychology. And within this paradigm of intensive motherhood, health is considered a private matter that the parents have to handle inside the family cocoon with limited exposure to the world at large. This, for the intensive mother, is the fundamental phase of nurturing and balance.

Intensive parents tend to harbor suspiciousness towards physicians and pediatricians who fail to share their views; vigilance towards the medical world is consistent, so much so, that child vaccines are refused, just as home schooling is the preferred option. The home ambience is the preferred world, rather than the school environment, and this, the profound responsibility of intensive parents, that is, to construct an ambience of affectivity and expressivity, and not merely place the child within an environment.

Intensive parents develop their identity as parents through such health and educational choices. They take up a unique position outside of the classical system, and define new norms of postmodern childcare. If every parent is able to make his own choices, he/she becomes a social actor by his commitment into parenthood shaping destinies and identities. Childcare becomes a tool for identity assertion and self-assertion, that intensive parents use to distinguish themselves and become social actors. The politic, more than one of social assertion, is precisely of social and familial child-care and wellbeing. Intensive parents claim that they are unique, and free to make their own choices, but also that they belong to a new social class, which questions social order through active health choices.

The trend of intensive motherhood, or intensive parenting, creates new behavioral norms, and encourages parents from other social groups to do the same. This tendency progressively spread strengthened by the protest attitude towards health choices and the resurgence of the popular voice. The shaping of destiny, the ability to understand the medical world, the (at times) higher education level, give intensive mothers, and parents, a vivid thirst for applicable knowledge in the scope of self-management and identity assertion. This attitude is also to be found in other aspects of their lives, generating new social dynamics, by shaping new norms and new structures, especially where healthcare is concerned.

\section{Conclusion}

Intensive parents criticize modern medicine that is used in a systematic and feckless way, but they do not reject it as a whole, since they acknowledge its usefulness in pathological cases and health crisis. They do not disapprove wholly of institutionalized medicine but sooner seek to make it their own in processes of adaption and redefinition. These statements are true for healthcare in general, but intensive parents' attitudes are even more asserted when it comes to pregnancy, childbirth, and child health.

Intensive parents question the institutionalization of maternity, and state control: they sooner favor the private sphere. By staying on the fringe of the institutional healthcare system, they manage to assert as individuals, and as couples first, and later, as a new social group. Self assertion is generated by alternative ideas translated into a defense attitude, which is used by the group and the individuals as energy to assert their unique (health and social) choices. "Birth is a special moment in family life, they are the actors of the birth." (Jacques, 2007, p. 177)

It is nowadays harder to become a parent, since birth is no longer surrounded by tradition. The processes of becoming a parent entails personal history and individual potential, because, as underlined by Christine Castelain-Meunier (2002, p. 143), "birth conception is nowadays a private matter; it affects the parents to be in their individuality". People no longer want to pass over the values of a special social group, but sooner seek to impart their own identity patrimony. These changes constitute parenthood to be an individual experience: giving birth to a child is nowadays generally a matter of choice that people feel responsible for, by considering births as personal events. 
Parents have to make several practices their own, in order to show their abilities as mothers and fathers, and in order to assert their cultural choices, their involvement, and their responsibilities towards the chosen models (Dubet, 1998).

The development of subjectivity and individuality generates a complex culture of parenthood. The changes in parental functions imply a symbolic, social, cultural, and ideological questioning, which call upon the responsibility of state, institutions, associations, and the whole civil society (Castelain-Meunier, 2002; Supiot, 2001). According to Michel Wieviorka (1998, p. 215), "Commitment is from now on increasingly experienced as requesting a strong coherence between general, political, social, cultural aspirations, that give a meaning to the action and the place that the latter have in personal experience".

Intensive motherhood intends to step away from technical healthcare practices, and thus questions the way individuality and subjectivity is handled by medicine; it enables us to develop better analyses about pregnancy and labor care. The whole French healthcare system is questioned in order to be improved. We are moving on from the era of pure technique to an era of (re)humanization. Even though medical technique is taken for granted; we should keep these tools as a basis to be developed: regulating them should help people make every labor unique, every pregnancy and maternity special, and each individual truly the master of their own identity.

The health paths of intensive mothers show the intimate link between health and identity. Health defines the human being and health choices mark social choices even though at times these choices may be at variance with the establishment. The ethos of such a philosophy is that a newborn's health exerts the parental will in the shaping of the current world, where an actor of health choices, is simultaneously an actor of society.

\section{Bibliography}

Badinter E. (2010) Le conflit, la femme et la mère, Flammarion, Paris.

Blondel, B., Kermarrec, M. (October 2011). La situation périnatale en France en 2010. DREES, U953 INSERM, N 775 , Paris.

Brooks, D.(2000) Les bobos. Florent-Massot, Paris.

Bydlowsk, M. (2005). La dette de vie. PUF, Paris.

Canuel, B. (2007). Les enfants d'aujourd'hui. Bayard, Paris.

Castelain-Meunier, C.(2004) La place des hommes et les métamorphoses de la famille, PUF, Paris.

European Commission.(30/09/2004). EU Research on Social Sciences and Humanities, The Rational of Motherhood Choices: Influence of Employment Conditions and of Public Policies. Bruxelles.

Damon, J.(2006). Les Politiques Familiales, PUF, Paris.

Douglas, S.(2004). The Mommy Myth. The idealization of Motherhood. Free Press, New York.

Dubet, F. (1998). Dans quelle société vivons-nous ? Le Seuil, Paris.

Fagnani J. (2000) Un travail et des enfants, Bayard éditions, Paris

Faircloth, C. (February 2009). "Mothering as Identity-Work”, in Anthropology News, p. 15-17.

Gaborit, P.(2009). Les stéréotypes de genre, L’Harmattan, Paris.

Hattery, A.(2001). Women, work and Family. Balancing and Weaving, Sage Publications, California. 
Hays, S.(1998). The cultural contradictions of Motherhood. Yale University Press, New Haven.

Hewlett, SA.(2002). Baby Hunger - the new battle for motherhood. Atlantic Books, London.

Jacques, B.(2007). Sociologie de l'accouchement. PUF, Paris.

Kreyenfeld, H.(2004). Familiensoziologie, Lehrbuch. Hamburg.

Kniebiehler Y.(2000). Histoire des mères et de la maternité en Occident. PUF, Paris.

Margain, C.(2007). La femme au foyer est-elle l'avenir du féminisme ?, Calmann-Lévy. Paris.

Martuccelli, D.(2003). L’enfant chef de la famille. Albin Michel, 2003.

Rich A.(1995). Of Woman Born. Motherhood as Experience and Institution. W. W. Norton \& Company; Norton Pbk. Ed.

Segalen, M.(1995). La Famille en Europe, La découverte, Paris.

Silvera, R.(2002). Articuler vie familiale et vie professionnelle en Europe, La Documentation Française, Paris.

Supiot, A.(2001). Pour une politique des sciences de l'homme et de la société. PUF, Paris.

Touraine, A.(2006). Le monde des femmes, Fayard, Paris.

White H.(1992) Identity and Control : A Structural Theory of Social Action, Princeton University Press, Princeton.

World Health Organization.(2003). Managing complications in Pregnancy and Childbirth: A guide for midwives and doctors. WHO Library Cataloguing-in-Publication Data, 2003.

Wieviorka, M.(1998). Raison et conviction : l'engagement. Textuel, Paris.

Wieviorka, M.(2008). Neuf leçons de sociologie, Robert Laffont, Paris.

The Identity Work and Health of Intensive Motherhood 